\title{
EFFECTS OF IN VITRO 1,25 DIHYDROXYVITAMIN D ON MATURATION OF DENDRITIC CELLS IN GRAVES' DISEASE PATIENTS
}

\section{DYAH PURNAMASARI ${ }^{*}$, SAMSURIDJAL DJAUZI ${ }^{2}$, SITI SETIATI ${ }^{3}$, ALIDA HARAHAP ${ }^{4}$, TJOKORDA GDE PEMAYUN ${ }^{5}$ JOEDO PRIHARTONO ${ }^{6}$, PRADANA SOEWONDO ${ }^{1}$}

Department of Medicine, Division of Endocrinology, Faculty of Medicine University of Indonesia, Jakarta, DKI Jakarta Indonesia. ${ }^{2}$ Department of Medicine, Division of Allergy Immunology, Faculty of Medicine University of Indonesia, Jakarta, DKI Jakarta, Indonesia. ${ }^{3}$ Department of Medicine, Division of Geriatrics, Faculty of Medicine University of Indonesia, Jakarta, DKI Jakarta, Indonesia. ${ }^{4}$ Departement of Clinical Pathology, Division of Immunology, Faculty of Medicine University of Indonesia, Jakarta, DKI Jakarta, Indonesia. ${ }^{5}$ Department of Medicine, Division of Endocrinology, Faculty of Medicine Diponegoro University, Semarang, Central Java, Indonesia. ${ }^{6}$ Department of Community Medicine, Faculty of Medicine University of Indonesia, Jakarta, DKI Jakarta, Indonesia. Email: dyah_p_irawan@yahoo.com.

Received: 06 June 2016, Revised and Accepted: 08 June 2016

\section{ABSTRACT}

Objective: The autoimmune reaction in Graves' disease (GD) is induced by self-antigen, which is presented by dendritic cells (DCs). DCs in GD have more active immune responses than those in healthy subjects. The ability of DC as antigen-presenting cell is determined by its maturity level. In GD, vitamin D level is inversely proportional to antibody titer and proportionally associated with remission status. Studies on healthy subjects and autoimmune patients (systemic lupus erythematosus (SLE), multiple sclerosis (MS), and Crohn's disease) have demonstrated immunoregulatory effects of vitamin D, mainly through inhibition of DC maturation, which may decrease the DC's immunogenic profile. This study aims to identify the effect of 1,25-D3 in vitro on DC maturation in patients with GD.

Methods: This is an experimental study, which was conducted in 12 GD patients with thyrotoxicosis. Monocyte-derived DC of GD patients was cultured, with or without 1,25-D3 in vitro at monocytic phase. The DC maturation was then stimulated by lipopolysaccharide (LPS) and evaluated based on the expression of DC markers (human leukocyte antigen-D-related [HLA-DR], CD80, CD40, CD83, CD14, and CD206) and the ratio of cytokine interleukin-12 (IL-12)/IL-10 levels in the supernatants.

Results: Following the LPS stimulation, DC with 1,25-D3 showed lower expressions of HLA-DR, CD80, CD40, and CD83, and higher expressions of CD14 and CD206 compared to DC without 1,25-D3. DC with 1,25-D3 had lower ratio of IL-12/IL-10 levels than those without 1,25-D3.

Conclusion: In vitro 1,25-D3 supplementation inhibits DC maturation in patients with GD.

Keywords: Vitamin D, Graves' disease, Dendritic cells.

(C) 2016 The Authors. Published by Innovare Academic Sciences Pvt Ltd. This is an open access article under the CC BY license (http://creativecommons. org/licenses/by/4. 0/) DOI: http://dx.doi.org/10.22159/ajpcr.2016.v9i5.13287

\section{INTRODUCTION}

Graves' disease (GD), the most common cause of hyperthyroidism, is an autoimmune disease which is characterized by the presence of autoantibody (thyroid receptor antibody [TRAb]). In GD, inflammatory cytokines also increase, in which the interleukin-12 (IL-12) is the major one related to maturation and activation of dendritic cells (DCs) $[1,2]$. Mature DCs are potent antigen-presenting cells (APCs) and will induce an adaptive immune response to produce autoantibody.

DCs, especially intrathyroidal, are more immunogenic in GD than in healthy subjects [3]. The number and activity of DC are influenced by thyroid hormone (triiodothyronine [T3] and thyroxine[T4]) [4]; therefore, thyroid hormone will increase $\mathrm{DC}$ activity and vice versa, which results in continuous adaptive immune response.

In autoimmune disease, the level of DC maturation has an essential role since it determines its immunogenic properties. Mature DCs act as strong APCs, which are able to expose self-antigen to naïve T-cells and stimulate differentiation of T-cells. Immature DC (iDC) has the main capacity to capture and process antigen, but they are weak APC Mature DC produces a high level of pro-inflammatory cytokine IL-12 and low level of anti-inflammatory IL-10 [5]. DC maturation is affected by genetic and environmental factors. One of the environmental factors which suppress DC maturation is vitamin D [6].
In association with GD, both experimental and epidemiological studies have shown an association between vitamin D deficiency, vitamin $\mathrm{D}$ receptor, and vitamin $\mathrm{D}$ binding protein polymorphisms with the development of GD $[7,8]$. Clinically, vitamin D level is related with remission status [9] and inversely related to the TRAb titer in patients with GD [10]. In GD patients, vitamin D analog supplementation (1a-OH) together with antithyroid drugs (ATDs) reduce thyroid hormone level faster than those who receive ATD alone [11]. The reduction of thyroid hormone level is due to Vitamin D-induced alteration of thyroid hormone synthesis, while the immunomodulatory effect of vitamin D on the reduction of thyroid hormone level has not been studied yet.

In term of autoimmune process, inhibition of DC maturation is the main target of vitamin D supplementation. Studies in healthy subjects and those with autoimmune diseases (systemic lupus erythematosus [SLE], multiple sclerosis [MS], and Crohn's disease) demonstrate that vitamin D inhibits DC maturation in monocyte-derived DC (MDDC) cultures [12-15]. This study wants to know the effect of in vitro 1,25-D3 supplementation on DC maturation in patients with GD.

\section{METHODS}

Design

We enrolled 12 GD patients with thyrotoxicosis at the endocrinology clinic of Dr. Cipto Mangunkusumo Hospital in Jakarta, Indonesia. The diagnosis of GD was established based on clinical manifestations 
(tachycardia, fine tremor, excessive sweat, diffuse struma, and ophthalmopathy) and laboratory data (low thyroid-stimulating hormone [TSH] level, increased fT4 level, and positive TRAb). Patients who took vitamin D supplementation, pregnant, or having comorbidities (diabetes mellitus, chronic kidney disease, and other autoimmune diseases) were excluded from the study. This study has been approved by The Ethics of Committee of the Faculty of Medicine, Universitas Indonesia no.489/H2.F1/ETIK/2014. To be included in the study, subjects had to sign a written informed consent form.

Right after $30 \mathrm{ml}$ venous blood drawing, MDDC cultures were performed with two interventions: Without 1,25-D3 and with $100 \mathrm{nM} 1,25$-D3 at monocytic phases. MDDC cultures, which consisted of peripheral blood mononuclear cell (PBMC) isolation, monocytes isolation, and lipopolysaccharide (LPS) stimulation, were performed according to study protocol conducted by Budiyati [16]. Some blood specimens were centrifuged, and the serum was stored at $-80^{\circ} \mathrm{C}$ for hematologic analysis. Supernatants were stored at $-20^{\circ} \mathrm{C}$ before IL-12 and IL-10 evaluations.

\section{Measurement}

TSH, fT4, and TRAb levels were measured using commercial kits as follows: TRAb (DRG), TSH, and fT4 (ROCHE).

\section{MDDC culture: Mo-DC differentiation}

The PBMC isolation was performed by the method of density differences using Ficoll-Paque premium (GE health care), and the specimens were suspended in the Roswell Park Memorial Institute (RPMI) medium at cell density of $1 \times 10^{6} / \mathrm{mL}$. In the 12 -well culture plate, isolation of monocytes was performed using the adherence method. After the monocytes adhered to the bottom of plate, we added $1 \mathrm{~mL}$ of DC culture media, which was a mixture of RPMI 1640 medium, 10\% fetal bovine serum (FBS, Gibco), $800 \mathrm{U} / \mathrm{mL}$ of granulocyte-macrophage colonystimulating factor (BD Bioscience Pharmingen), and $1000 \mathrm{U} / \mathrm{mL}$ of IL-4 (BD Bioscience Pharmingen). The culture plate was then incubated at $37^{\circ} \mathrm{C}$ with $\mathrm{CO}_{2} 5 \%$ for 5 days.

\section{1,25-D3 stimulation}

The powder of 1,25-D3 (Sigma-Aldrich) was dissolved in 95\% ethanol and a stock solution was made at the concentration of $1 \mathrm{M}$ and was stored at $-80^{\circ} \mathrm{C}$. Prior to use, the stock solution of 1,25 -D 3 was diluted until the concentration of $100 \mathrm{nM}$ was reached.

\section{MDDC culture: DC maturation and harvesting}

After being incubated for 5 days, 500 ng LPS was added (SigmaAldrich) and incubation was continued for 2 days. Cell harvesting was performed twice, i.e., before adding LPS (D5) and after being incubated for 2 days with LPS (D7). Cell harvesting was done by light spray at the bottom of the wells, and the DC solution was then processed to separate supernatant from cells. The cells can be processed for monoclonal antibody $(\mathrm{mAb})$ staining.

\section{Flow cytometry analysis}

The fluorochrome-conjugated antibodies used in flow cytometry technique were obtained from BD Biosciences Pharmingen with the following details: Human leukocyte antigen-D-related (HLA-DR) fluorescein isothiocyanate (FITC) (G46-6, mouse IgG2ak); CD40 phycoerythrin (PE) (5C3, mouse IgG1א); CD80 PE-Cyanine5 (PECy5) (L307,4, mouse IgG1א); CD83 PECy5 (HB15e, mouse IgG1א); CD206 PE $(19,2$, mouse IgG1א), and CD14 FITC (M5E2, mouse IgG2ak). The flow cytometry analysis was performed using FACSCalibur (BD Biosciences).

The expressions of HLA-DR, CD80, CD40 and CD206 were measured in the form of mean fluorescence intensities; meanwhile the expressions of CD14 and CD83 were presented in numbers of positive cells.

Enzyme-linked immunosorbent assay (ELISA) analysis Cytokine levels (IL-12 and IL-10) in supernatant were measured using human IL-12 p70 Quantikine HS ELISA Kit - R\&D Systems kit and human
IL-10 Quantikine ELISA Kit - R\&D Systems according to instruction in the kit manual.

\section{Statistical analysis}

Data were presented in mean value \pm standard deviation when they were normally distributed and in median value (minimum and maximum value) when they were not normally distributed. The comparison of mean or median value for DC marker expression and ratio of IL-12/ IL-10 cytokines of DC between those with and without 1,25-D3 treatment was conducted using paired t-test when the data had normal distribution and Wilcoxon test when the data did not have a normal distribution.

\section{RESULTS}

Subject characteristics can be seen in Table 1.

Effects of in vitro 1,25-D3 on the expression of DC markers and the ratio of cytokines IL-12/IL-10 in MDDC cultures of GD patients

Effects of in vitro 1,25-D3 supplementation on DC maturation were observed in the cultures on the $5^{\text {th }}$ and $7^{\text {th }}$ day. On the $7^{\text {th }}$ day, the expressions of HLA-DR, CD80, CD40, and CD83 in the cultures with 1,25 -D3 were lower than those cultures without 1,25 -D3 ( $p=0.016$; $p<0.01 ; p=0.056 ; p=0.019)$; the expression of CD14 in the cultures with 1,25 -D3 was higher than those cultures without $1,25-\mathrm{D} 3(\mathrm{p}<0.01)$, while the expression of CD206 in the cultures with 1,25-D3 was maintained $(p=0.3375)$ (Fig. 1).

On the $5^{\text {th }}$ day, the expression of HLA-DR in the cultures with 1,25-D3 was lower than the cultures without $1,25-\mathrm{D} 3(\mathrm{p}<0.01)$, while the expression of CD14 in the cultures with 1,25-D3 was higher than the cultures without 1,25-D3 (p<0.01) (Fig. 2).

On the $5^{\text {th }}$ day of culture, 1,25-D3 supplementation did not change the expression of CD206 ( $\mathrm{p}=0.3375)$. The ratio of IL-12/IL-10 in the cultures with 1,25-D3 was significantly lower than those cultures without 1,25-D3 (Fig. 3).

\section{DISCUSSION}

As a natural immunomodulator, vitamin D increases innate immune response and regulates excessive adaptive immune response such as found in autoimmune disease [17]. DCs are immune cells that initiate and maintain the autoimmune response [5]. One of the important effects of vitamin D in autoimmune is inhibition of DC maturation. The present study provides additional information regarding the immunoregulator effect of vitamin D, particularly on inhibition of DC maturation in GD patients. This is the first study reporting the effect of in vitro vitamin D on inhibition of DC maturation in GD.

Effects of in vitro 1,25-D3 on the expression of DC markers in MDDC cultures of GD patients

This study shows that in vitro 1,25-D3 supplementation inhibits DC maturation in MDDC cultures of GD patients, which is characterized by lower expression of major histocompatibility complex class II

Table 1: Subject characteristics $(n=12)$

\begin{tabular}{lll}
\hline Characteristics & $\begin{array}{l}\text { Mean } \\
\text { value } \pm \text { SD }\end{array}$ & $\begin{array}{l}\text { Median } \\
\text { (minimum-maximum) }\end{array}$ \\
\hline $\begin{array}{l}\text { Age (years) } \\
\text { Duration of illness } \\
\text { (months) }\end{array}$ & $35.83 \pm 10.74$ & $20(2-204)$ \\
TSH $(\mu \mathrm{IU} / \mathrm{L})$ & 0.01 & \\
fT4 $(\mathrm{ng} / \mathrm{dL})$ & & $6.61(2.69-7.77)$ \\
TRAb (U/L) & $28.17 \pm 25.33$ & \\
Vit 25-D3 (ng/mL) & $26.99 \pm 6.89$ & - \\
\hline
\end{tabular}

Data are presented in mean value \pm SD when they were normally distributed and in median value (minimum and maximum value) when they did not have normal distribution. SD: Standard deviation, TSH: Thyroid stimulating hormone, TRAb: Thyroid receptor antibody 

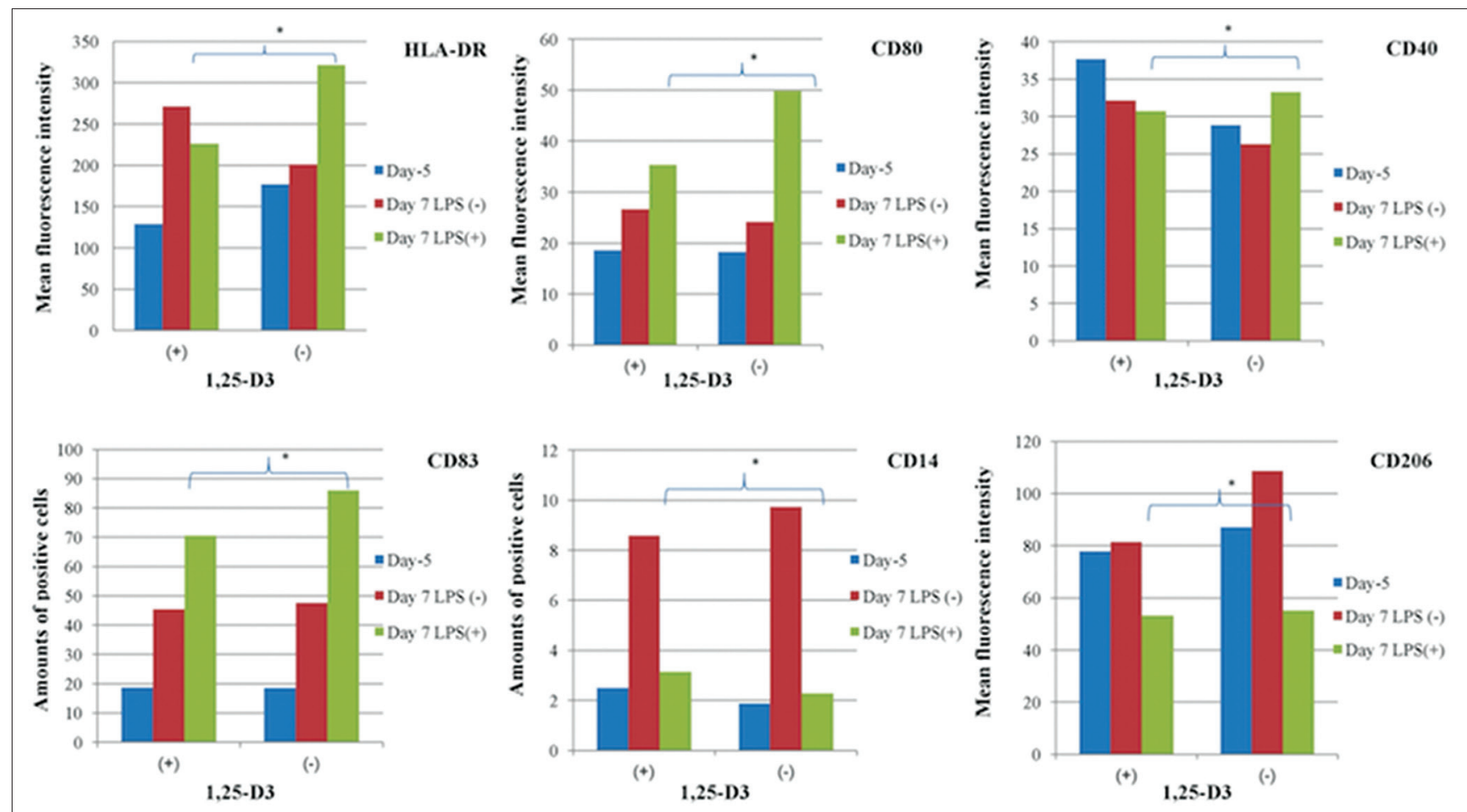

Fig. 1: The expressions of DC molecules in cultures day 7. *The expressions of DC molecules on day 7 (LPS+) in cultures without and with $1,25-D 3, n=12$

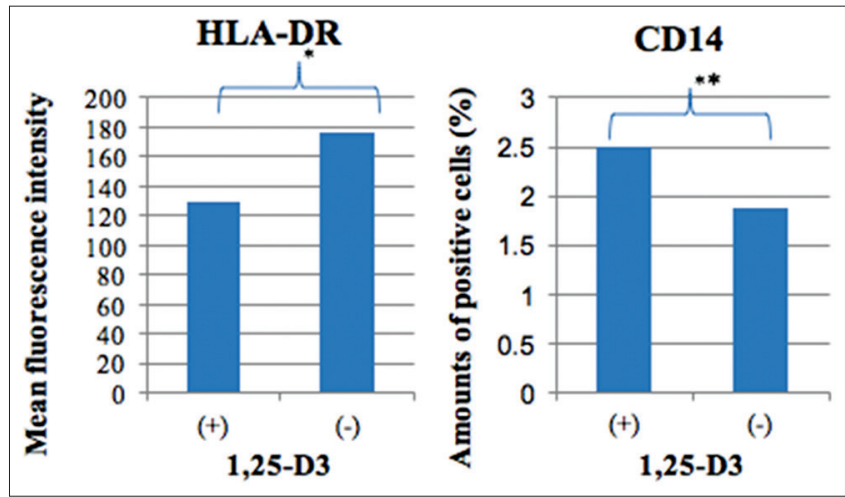

Fig. 2: The expressions of human leukocyte antigen-D-related (HLA-DR) and CD14 in cultures day 5. *The expression of HLA-DR in cultures day 5 with and without $1,25-\mathrm{D} 3$ in vitro $(\mathrm{p}<0.05$, paired $t$-test), $\mathrm{n}=12$. ${ }^{* *}$ The expression of CD14 in cultures day 5 with and without $1,25-\mathrm{D} 3$ in vitro ( $p<0.05$, Wilcoxon test), $n=12$

(HLA-DR) molecules, costimulatory molecules (CD80 and CD40), and marker of mature DC (CD83) $(\mathrm{p}<0.05)$. Any disruption on DC maturation may result in weak immunogenicity of DC, which may lead to lower DC ability as APC and activator of naïve T-cells. The in vitro 1,25-D3 supplementation also increases monocyte marker (CD14) and maintain MR expression (CD206), which are major markers for monocytes and iDC; therefore, it demonstrates the capacity of 1,25-D3 to maintain DC immaturity status. Those results are consistent with other experimental studies among healthy subjects, SLE, and Crohn's disease $[12,14]$.

Other findings in our study are the change of DC molecules expression on the $5^{\text {th }}$ day. At iDC phase, the expressions of HLA-DR and CD14 have already changed but other markers have not. In vitro 1,25-D3 in monocytic phase reduced the expression of HLA-DR $(\mathrm{p}<0.01)$ and increased the expression of CD14 $(p<0.01)$ of iDC on the $5^{\text {th }}$ day, while

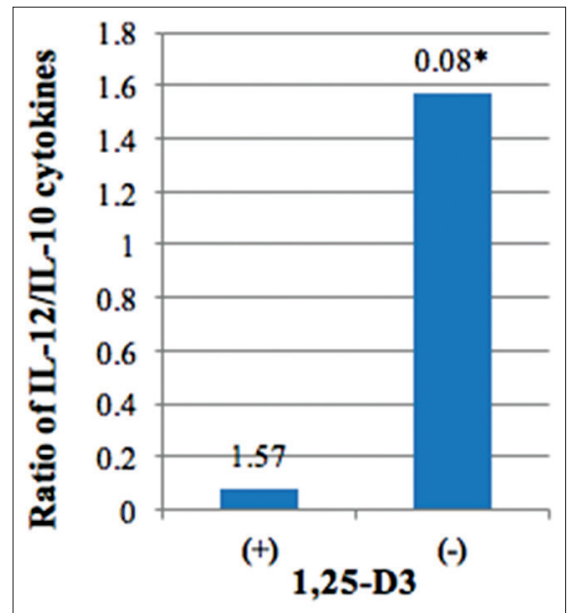

Fig. 3: The ratio of interleukin-12 (IL-12)/IL-10 in cultures with and without 1,25-D3. ${ }^{*} \mathrm{p}<0.01$, Wilcoxon test, $\mathrm{n}=12$

the expressions of CD80, CD40, CD83, and CD206 showed no difference $(\mathrm{p}>0.05)$.

HLA-DR molecule is very important since polymorphism or genetic mutation of HLA-DR has a role in the pathogenesis of some autoimmune diseases including GD $[18,19]$. The complex HLA-DR gene is the major genetic factor in AITD in addition to the gene of Treg cells (CTLA4) and specific genes of thyroid [19]. It explains why the expression of HLA-DR in MDDC culture has been affected since the iDC phase by in vitro 1,25-D3 supplementation, while the expression of CD80, CD40, CD83, and CD206 was not affected. The role of CD14 in GD still cannot be explained as clearly as the role of HLA-DR. The change of CD14 expression is earlier and probably due to the essential role of the molecule in the GD progression. It needs further studies considering that there is still no study reporting the correlation between the genetic defect of CD14 molecule and GD. 
Effects of in vitro 1,25-D3 on the ratio of cytokines IL-12/IL-10 levels in MDDC cultures of GD patients

The balance between cytokines IL-12 and IL-10 is required to maintain tolerance [20]. This study used IL-12/IL-10 ratio to provide a better description of both cytokines interaction [20]. It shows that in vitro vitamin D supplementation in MDDC cultures of GD patients decreases the ratio of IL-12/IL-10 cytokines. This is the first study which demonstrates the effect of in vitro 1,25-D3 supplementation on IL-12/IL-10 ratio in patients with autoimmune disease. Lower IL-12/IL-10 ratio suggests lower DC immunogenicity.

If each cytokine is evaluated separately in this study, in vitro 1,25-D3 supplementation decreases IL-12 cytokine level significantly, while IL-10 cytokine level is relatively maintained. It indicates that in vitro 1,25-D3 supplementation can suppress IL-12 production and maintain the role of IL-10 on immune response initiated by DC. Low inflammatory cytokines level will reduce T-cells activation leading to prevention of persistent immune response in autoimmune cases $[5,20]$.

The effect of in vitro vitamin D supplementation on IL-12 or IL-10 cytokines has been previously reported for SLE, MS, and Crohn's disease. Studies on Crohn's disease did not demonstrate the effect of in vitro vitamin D supplementation on the decrease of IL-12 level because they used less sensitive ELISA kit. Studies on SLE and MS only evaluated either IL-12 or IL-10, therefore, may not provide balanced description of inflammatory and anti-inflammatory cytokines produced by DC $[12,13]$.

\section{CONCLUSION}

In vitro 1,25-D3 supplementation inhibits DC maturation in patients with GD.

\section{ACKNOWLEDGMENTS}

Author wants to acknowledge DIKTI (The Directorate General of Higher Education) Research Grants 2014 program for grants given to support this research. The content is solely the responsibility of authors and does not necessarily represent general view of the University of Indonesia.

\section{REFERENCES}

1. Tamura M, Matsuura B, Miyauchi S, Onji M. Dendritic cells produce interleukin-12 in hyperthyroid mice. Eur J Endocrinol 1999;141(6):625-9.

2. Tamaru M, Matsuura B, Onji M. Increased levels of serum interleukin-12 in Graves' disease. Eur J Endocrinol 1999;141(2):111-6.

3. Vasu C, Holterman MJ, Prabhakar BS. Modulation of dendritic cell function and cytokine production to prevent thyroid autoimmunity. Autoimmunity 2003;36(6-7):389-96.
4. Dedecjus M, Stasiolek M, Brzezinski J, Selmaj K, Lewinski A. Thyroid hormones influence human dendritic cells' phenotype, function, and subsets distribution. Thyroid 2011(5);21:533-40.

5. Banchereau J, Steinman RM. Dendritic cells and the control of immunity. Nature 1998;392(6673):245-52.

6. Baeke F, Takiishi T, Korf H, Gysemans C, Mathieu C. Vitamin D: Modulator of the immune system. Curr Opin Pharmacol 2010;10(4):482-96.

7. Pani MA, Regulla K, Segni M, Hofmann S, Hüfner M, Pasquino AM, et al. A polymorphism within the Vitamin D-binding protein gene is associated with Graves' disease but not with Hashimoto's thyroiditis. J Clin Endocrinol Metab 2002;87(6):2564-7.

8. Zhou H, Xu C, Gu M. Vitamin D receptor (VDR) gene polymorphisms and Graves' disease: A meta-analysis. Clin Endocrinol (Oxf) 2009;70(6):938-45.

9. Yasuda T, Okamoto Y, Hamada N, Miyashita K, Takahara M, Sakamoto F, et al. Serum vitamin D levels are decreased in patients without remission of Graves' disease. Endocrine 2013;43(1):230-2.

10. Zhang H, Liang L, Xie Z. Low Vitamin D status is associated with increased thyrotropin-receptor antibody titer in Graves disease. Endocr Pract 2015;21(3):258-63.

11. Kawakami-Tani T, Fukawa E, Tanaka H, Abe Y, Makino I. Effect of 1 alpha-hydroxyvitamin D3 on serum levels of thyroid hormones in hyperthyroid patients with untreated Graves' disease. Metabolism 1997;46(10):1184-8.

12. Wahono CS, Rusmini H, Soelistyoningsih D, Hakim R, Handono K, Endharti AT, et al. Effects of 1,25(OH)2D3 in immune response regulation of systemic lupus erithematosus (SLE) patient with hypovitamin D. Int J Clin Exp Med 2014;7(1):22-31.

13. Bartosik-Psujek H, Tabarkiewicz J, Pocinska K, Stelmasiak Z, Rolinski J. Immunomodulatory effects of vitamin D on monocyte-derived dendritic cells in multiple sclerosis. Mult Scler 2010;16(12):1513-6.

14. Bartels LE, Jørgensen SP, Bendix M, Hvas CL, Agnholt J, Agger R, et al. 25-Hydroxy vitamin D3 modulates dendritic cell phenotype and function in Crohn's disease. Inflammopharmacology 2013;21(2):177-86

15. Berer A, Stöckl J, Majdic O, Wagner T, Kollars M, Lechner K, et al. 1,25-Dihydroxyvitamin $\mathrm{D}(3)$ inhibits dendritic cell differentiation and maturation in vitro. Exp Hematol 2000;28(5):575-83.

16. Budiyati AD, Setiyono A, Tarigan E, Wibowo $\mathrm{H}$. The effect of alpha fetoprotein on NF-kB translocation in lipopolysaccharide induced monocyte-derived dendritic cell. Med J Indones 2012;21(2):1-5.

17. Hewison M. Vitamin D and the immune system: New perspectives on an old theme. Endocrinol Metab Clin North Am 2010;39(2):365-79.

18. Park MH, Park YJ, Song EY, Park H, Kim TY, Park DJ, et al. Association of HLA-DR and - DQ genes with Graves disease in Koreans. Hum Immunol 2005;66(6):741-7.

19. Swain M, Swain T, Mohanty BK. Autoimmune thyroid disorders-An update. Indian J Clin Biochem 2005;20(1):9-17.

20. Jiang HR, Muckersie E, Robertson M, Xu H, Liversidge J, Forrester JV. Secretion of interleukin-10 or interleukin-12 by LPS-activated dendritic cells is critically dependent on time of stimulus relative to initiation of purified DC culture. J Leukoc Biol 2002;72(5):978-85. 